\title{
DYNAMIC SPECKLE CORRELATIONS
}

\author{
Frank Scheffold \\ Fakultät für Physik, Universität Konstanz, Postfach 5560, D-78547 Konstanz, Germany \\ Physikalisches Institut, Universtät Freiburg, CH-1700 Freiburg, Switzerland \\ Frank.Scheffold@unifr.ch \\ Georg Maret \\ Fakultät für Physik, Universität Konstanz, Postfach 5560, D-78547 Konstanz, Germany \\ Georg.Maret@uni-konstanz.de
}

\begin{abstract}
Long- and infinit range correlations $C_{2}$ and $C_{3}$ in the optical speckle pattern represent one of the most interesting phenomena in multiple scattering of light. Despite the strong scattering these correlations survive the averaging process of light diffusion and are even enhanced with increased randomness. In this article we are going to discuss the microscopic origin of these particular correlations which are explained in the simple picture of one and twofold crossing of multiple scattering paths. We present a comprehensive experimental study of dynamic speckle correlations, $C_{2}(t)$ and $C_{3}(t)$, where the phase shift between the multiple scattering paths is caused by the Brownian motion of the scattering particles. The shape and amplitude of the correlation functions $C_{2}(t)$ and $C_{3}(t)$ are in good overall agreement with theory. Deviations are found in the case of $C_{2}(t)$ when correlations are generated close to the incoming surface which can be explained by single scattering contributions.
\end{abstract}

Keywords: speckle correlations, diffusing wave spectroscopy, conductance fluctuation

\section{INTRODUCTION}

Light propagation in random media has attracted considerable attention over the last decade. In analogy to electronic transport in disordered metals, fundamental issues such as localization of light have been addressed [1,2]. It has been found that despite the randomness of the medium, various interference effects are essential for the light propagation in the multiple scattering regime. 
Since the discovery of weak localization of light [3, 4], a precursor of light localization, a much deeper understanding on wave propagation in random media has been achieved $[5,6,7]$. Most recently much attention has been paid to the reported observation of strong localization of light, which has been discussed controversially $[8,9,10]$.

Another most interesting phenomenon in multiple scattering of classical waves is the appearance of correlations and fluctuation in the transmission speckle pattern. In this article we discuss recent experimental results about these correlations in the dynamic speckle pattern of laser light transmitted through a turbid colloidal suspension. Due to particular interference effects caused by crossing of scattering paths inside the random medium, two types of correlations between different speckle spots build up $[11,12,13,14,15,16,17,18,19]$. (1) Long range correlations in the scattered field give rise to fluctuation in the angular integrated transmission. (2) Infinit range correlations cause fluctuation in the total transmission, independent both of the incoming and transmitted wave mode. The latter fluctuation are considered the optical analogue of "universal conductance fluctuation (UCF)" in electronic systems [13, 20].

After a brief review of the physical origin and theory, we discuss the temporal shape of the correlation function $C_{2}(t)$ and its amplitude dependence on sample thickness, beam spot size, and transport mean free path $l^{*}$. Universal conductance fluctuation of light will be the subject of the fina part of this article.

The experiments show that due to of the inherently small noise level in dynamic light scattering experiments, photon correlation spectroscopy provides access to an unprecedented accuracy in the study of optical speckle correlation phenomena.

\section{THEORY}

\subsection{THE PHYSICAL PICTURE}

Both classical and electronic conductance fluctuation can be described in an appealing simple physical picture (Fig. 1.1) as further outlined below $[13,18$, 19]. $\left(C_{1}\right)$ : Interferences between waves scattered along independent paths give rise to short range angular fluctuations in optics known as speckles. These are due to non-intersecting scattering paths which give rise to short range temporal and angular speckle fluctuation because of scatterers motion. There are no correlations between field scattered along different paths. $\left(G_{2}\right)$ : One crossing of scattering paths builds up correlations between different paths. Temporal decorrelation like in $\left(C_{1}\right)$ occurs along the active section of the paths located before the crossing event, while after the crossing the field remain totally correlated (no mutual phase shifts) at all $t$ and all output directions $(b, b)$. $\left(C_{3}\right)$ : Twofold crossings generate universal conductance fluctuation (UCF). 

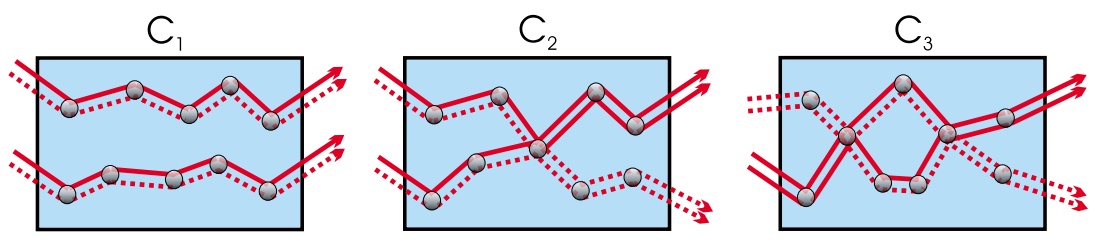

Figure 1.1 The physical origin of speckle correlations can be explained in an appealing simple physical picture of independent $\left(C_{1}\right)$ and crossing light paths $\left(C_{2}\right.$ and $\left.C_{3}\right)$. One crossing of paths generates correlations of all output speckle spots $\left(C_{2}\right)$ while two crossings cause UCF $\left(C_{3}\right)$. (-) wave field at correlation time $t=0,(--)^{2}$ phase shifted wave field at $t>0$ scattered along the same sequence of scatterers.

$t$-dependent phase shifts occur only between the crossing events, the intensity fluctuation are therefore insensitive to input $(a, d)$ and output $\left(b, b^{\prime}\right)$ wave modes.

\subsection{LONG AND INFINITE RANGE CORRELATIONS}

The cylindrical waveguide. The most simple case from a theoretical point of view is the diffuse transmission of classical waves through a cylindrical waveguide with perfectly reflectin walls (length $L$, width $D$ ). The average intensity, which is transmitted from an incoming plane light wave mode $a$ to an outgoing plane wave mode $b$, is called $\left\langle T_{a b}\right\rangle$. The (dimensionless) conductance $g$ of the sample is then define as the sum over all incoming and outgoing modes:

$$
g \equiv \sum_{a, b}\left\langle T_{a b}\right\rangle=\frac{N l^{*}}{L} \sim D^{2}
$$

$N$ is the number of modes inside the waveguide of length $L . N$ is proportional to $D^{2}$ and thus $g$ is proportional to the surface area of the sample.

Feng et. al. found that the intensity autocorrelation function $C(x)=$ $\langle I(0) I(x)\rangle^{2} /\langle I(0)\rangle^{2}-1$ can be written in terms of three leading contributions $C_{1}(x), C_{2}(x)$ and $C_{3}(x), x$ being some quantity, such as frequency shift $\Delta \omega$ or correlation time $t$, which introduces phase shifts between optical field $[11,13]$ :

$$
C(x)=C_{1}(x)+C_{2}(x)+C_{3}(x)
$$

The amplitude of the different contributions was found to scale with

$$
C_{1}(0) \simeq 1, C_{2}(0) \simeq g^{-1}, C_{3} \simeq g^{-2}
$$

In this respect $1 / g$ also describes the probability that two paths cross somewhere inside the sample [13]. Here $C_{2}=C_{2}(0)$ and $C_{3}=C_{3}(0)$ are indepen- 
dent of the functional behavior of $C_{2}(x)$ and $C_{3}(x)$, i.e. they are independent of the nature of $x$.

Slab geometry. In practice it is difficul to realize a small optical waveguide for diffuse light propagation with perfectly reflectin walls. Therefore already Feng et. al. suggested to investigate the transmission through a slab instead [11]. Pnini and Shapiro extended the theory of Feng to the general case of a finit beam spot incident on a slab [12]. They calculated the amplitude $G_{2}$ for a homogeneous beam spot of size $W$, with $I(r)=1$ for $0 \leq r<W / 2$ and $I(r)=0$ otherwise. The result for $W \gg L$ is the same as for a cylindrical waveguide while for the case $W<<L$ they fin that the amplitude scales linearly with the inverse beam spot size $1 / W[21]$ :

$$
\begin{aligned}
C_{2} & =\frac{4}{k_{0}^{2} W^{2}} \frac{L}{l^{*}} ; W \gg L \\
C_{2} & =\frac{3}{2 k_{0}^{2} W l^{*}} ; \quad W \ll L
\end{aligned}
$$

Later de Boer et. al. generalized this result for an incident gaussian beam, beamspot size $w$, usually encountered in optical experiments [22]. Although they considered frequency correlations $C(\Delta \omega)$ their results for the amplitude $C_{2}$ apply to the case of temporal correlations as well because of the insensitivity of $C_{2}$ on the phase shift introducing parameter:

$$
\begin{gathered}
C_{2}=\frac{1}{\alpha} \times \frac{L}{w^{2}} \times F\left(\frac{w}{L}\right) \\
\alpha=\frac{k_{0}^{2} l^{*}}{3}=\frac{l^{*}}{3}\left(\frac{2 \pi n}{\lambda}\right)^{2} \\
F\left(\frac{w}{L}\right)=\int_{0}^{\infty} d x\left(\frac{w}{L}\right)^{2} \exp \left[-\left(\frac{w}{L}\right)^{2} \frac{x^{2}}{32} \frac{x\left(\frac{\sinh (x)}{x}-1\right)}{8(\cosh (x)-1)}\right]
\end{gathered}
$$

where $n$ is the refractive index, and $\lambda$ is the wavelength of the incident light. In the limit $w>>, F(\infty) \longrightarrow 2 / 3$, hence $C_{2} \sim w^{-2}$.

\subsection{SHAPE OF THE CORRELATION FUNCTION}

Diffusing wave spectroscopy. Light transmission through a slab containing Brownian particles shows strong fluctuation in the transmission speckle 
pattern. The fluctuation of the individual speckle spots are determined by the autocorrelation of electromagnetic field scattered along individual paths, where the phase shift along a single path is, on average, directly proportional to the length of the path. For this case the leading contribution to the intensityintensity correlation function $\langle I(0) I(t)\rangle /\langle I(0)\rangle^{2}-1 \simeq C_{1}(t)$ can be derived from diffusion theory [23]. Assuming light propagation on independent scattering paths (Fig. 1.1), it is possible to calculate the actual distribution of light paths and therefore calculate $C_{1}(t)$ :

$$
C_{1}(t, L) \cong \exp \left(-2\left(\frac{L}{l^{*}}\right)^{2} \frac{t}{\tau_{0}}\right)
$$

It was shown experimentally that this relation holds very well for samples of thickness $L$ larger than 10 transport mean free paths $l^{*}$ [24]. Here $\tau_{0}=1 / D k_{0}^{2}$ denotes the single scattering decay time and $D_{0}$ the translational diffusion constant of the scatterers. The correlation function is dominated by a typical path length $L^{2} / l^{*}$ of the diffusing light where each scattering event contributes on average by $\exp \left(-t / \tau_{0}\right)$ to the decay of the correlation function. Measurements of $C_{1}(t)$ are widely exploited as the so called diffusing wave spectroscopy (DWS), which has become a powerful tool to study dynamics of colloids, emulsions, and other turbid soft matter $[23,25,26]$

Long range correlations. Unlike the amplitude $C_{2}$, the dynamical part of the long range correlations $C_{2}(\Delta \omega)$ cannot be directly transferred to the time domain $C_{2}(t)$. To our knowledge the only theoretical treatment of long range $C_{2}(t)$ correlations for Brownian scatterers is presented in a paper by Berkovits and Feng [14]. Using a diagrammatic technique they derive the intensityintensity correlation function for the case $w>>L$ and find

$$
\begin{aligned}
C_{2}(t) & =\frac{3 C_{2}}{2 \sqrt{6 \frac{t}{t_{0}}}}\left[\operatorname{coth}\left(\sqrt{6 \frac{t}{t_{0}}}\right)-\frac{\sqrt{6 \frac{t}{t_{0}}}}{\sinh ^{2}\left(\sqrt{6 \frac{t}{t_{0}}}\right)}\right] \\
t_{0} & \approx\left(\frac{l^{*}}{L}\right)^{2} \tau_{0}
\end{aligned}
$$

The $C_{2}(t)$ correlation function decays over a much broader time scale than in the case of short range $C_{1}(t)$ correlations. In the long time limit an algebraic $t^{-1 / 2}$ behavior is predicted. 


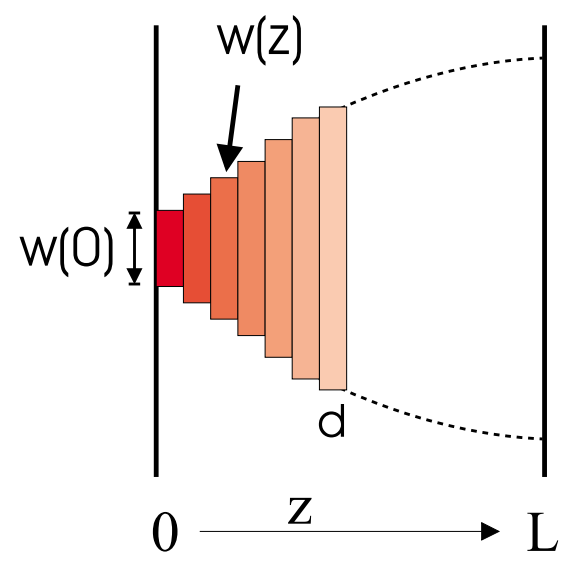

Figure 1.2 Broadening of the photon cloud for the slab geometry. The increasing width $w(z)$ leads to a sharp decline of the "crossing probability" $C_{2}(z)$ with increasing depth $z$. If the beam spot size $w<<L$, crossing events close to the incoming surface have a much higher weight and dominate $C_{2}$ and the temporal shape of the correlation function since the effective path lengths before a crossing event are much shorter as compared to a cylindrical waveguide of identical thickness $L$. With increasing beam spot size $w$ the decay of the crossing probability becomes less sharp until in the case $w>>$ it is independent of the depth $z$.

\subsection{THE INTEGRAL APPROXIMATION}

Most of the theoretical results described above are derived from diagrammatic calculations which are quite complicated and physically not always very instructive. Most of these calculations are restricted to ideal cases, like the cylindrical waveguide. Often however the theoretical assumptions do not match the experimental conditions, e.g. the sample geometry or the influenc of the boundary. How these deviations influenc the amplitude and the decay of $C(t)$ cannot be easily derived from standard theory without doing the whole calculation from scratch. On the other side we have seen that the physics of long and infinit range correlations can be understood within the simple picture of crossing light paths [section 2.1], where the crossing probability is of the order $C_{2} \approx 1 / g$. While in the cylindrical waveguide the crossing probability is the same throughout the sample this is not true for other geometries. Fig. 1.2 shows the conically shaped photon cloud in the case of a slab geometry. Here the crossing probability decreases with increasing depth $z$. Based on the simple picture of crossing light paths and the exact result for a cylindrical waveguide we have derived an approximate theory which can easily be adapted to the experimental conditions $[27,28]$. As a starting point we consider the sample as a succession of $\mathrm{Q}$ thin slabs $i$ of thickness $d[Q \cdot d=L]$. The thickness of the slabs is chosen such that $d$ is much smaller than the lateral extension $w(z)$ 


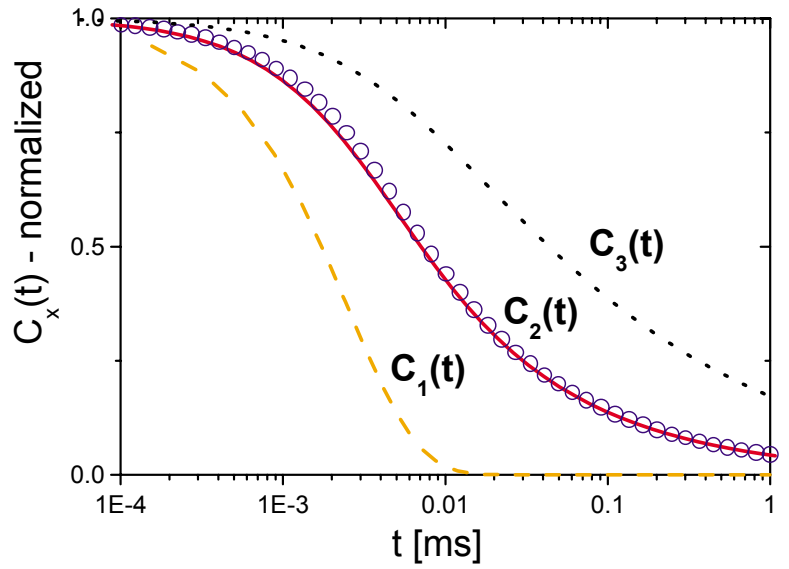

Figure 1.3 Comparison of the shape of the correlation functions $C_{1}(t), C_{2}(t)$ and $C_{3}(t)$ for $\left(L / l^{*}\right)=20, \tau_{0}=2 m s$. The correlated contributions $C_{2}(t)$ and $C_{3}(t)$, Eq. (1.16), decay slower and over a much broader time range than $C_{1}(t)$. A comparison of the diagrammatic results for $C_{2}(t)$ [Eq. (1.10), solid line] and the result obtained from the integral approximation [Eq. (1.15), open symbols] shows excellent agreement.

of the photon cloud at a depth $z$ inside the sample, hence $w(z) \gg d$. Each single slab can therefore be treated as a cylindrical waveguide with a crossing probability inside the slab $i$ [Eq. (1.4)]:

$$
C_{2}^{i} \propto \frac{d}{w(z)^{2}}
$$

From this we fnd for the amplitude:

$$
C_{2} \simeq \sum_{i=1}^{Q} C_{2}^{i} \stackrel{d \rightarrow 0}{\rightarrow} \frac{1}{L} \int_{0}^{L} C_{2}(z) d z
$$

It is straightforward to extend this expression to describe the decay of the correlation function as well. Contributions to $C_{2}$ which are due to a crossing of paths in a depth $z$ exhibit dephasing before the crossing event and are correlated afterwards. The path length distribution in this case is well approximated by the path length distribution of the uncorrelated function $C_{1}(t, z)$ [Eq. (1.9)]. We can therefore write:

$$
C_{2}(t)=\frac{1}{L} \int_{0}^{L} C_{2}(z) \cdot C_{1}(t, z) d z
$$


In the particular case of $C_{2}(z)=$ const. (cylindrical waveguide) we $\mathrm{f}$ nd the simple result:

$$
C_{2}(t)=C_{2} \frac{1}{L} \int_{0}^{L} C_{1}(t, z) d z
$$

If we compare this result with the exact result Eq. (1.10) from diagrammatic calculations, excellent agreement is found (Fig. 1.3). This demonstrates the consistency of our approach based on the simple physical picture described above. Eq. (1.14) represents a complete description of long range speckle correlations $C_{2}(t)$ for a known distribution of $w(z)$.

The same approach can be used to determine the correlation function $C_{3}(t)$ [29]. The active path sections contributing to $C_{3}(t)$ are located between two crossing events (Fig. 1.1) resulting in a further broadening and slowing down compared to $C_{2}(t)$. In the integral approximation the correlation function for a waveguide geometry is given by a double integral over $C_{1}(t, z)$ or a single integral over $C_{2}(t, z)$ :

$$
C_{3}(t)=\frac{C_{3}}{L} \int_{0}^{L} C_{2}(t, z) / C_{2} d z
$$

\section{DYNAMIC LONG RANGE CORRELATIONS}

\subsection{EXPERIMENT}

Dynamic long range correlations have been studied by angular averaging of light transmitted through a slab containing a turbid colloidal suspension. The colloidal suspensions were prepared from monodisperse $\mathrm{BaTiO}_{3}$ suspended in water [30]. Values of $l^{*}$ where determined independently by static transmission measurement [24]. A minimal value of $l^{*}=0.98 \mu \mathrm{m}$ was found at a volume fraction $\Phi=27 \%$. The fuctuations of the integrated transmission were measured with the setup illustrated in Fig. 1.4.

A gaussian laser beam (diameter roughly $1 \mathrm{~mm}$ ) from an Ar-laser operating in single frequency mode at $457.9 \mathrm{~nm}$ was focused onto a sample cell of variable thickness yielding a transverse intensity prof le at the sample surface :

$$
I(r)=\frac{2}{w^{2} \sqrt{\pi}} \exp \left(-\frac{4 r^{2}}{w^{2}}\right)
$$

The beam waist $w$ is def ned by the distance between the 1/e points of the transverse intensity distribution. To obtain small beam spot sizes, we used either an optical lens of a focal length of $5 \mathrm{~cm}$, which yields a minimum beam 


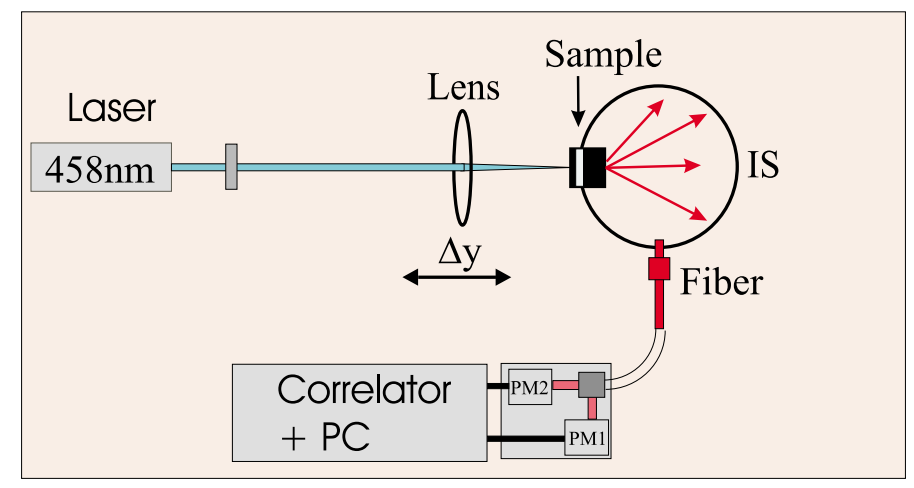

Figure 1.4 Experimental setup: The fuctuations of the integrated transmission through a slab were measured by angular averaging the transmitted light with an integrating sphere (IS). Detected by a photomultiplier unit (PM) the correlation function was subsequently analyzed using a digital correlator [18]. The incident laser beam is strongly focused by a lens.

spot size of $w=11.6 \mu \mathrm{m}$, or in one case a microscope objective to obtain beam spots down to $w=3.4 \mu \mathrm{m}$. This setup allowed us to change the actual beam spot size by variation of the sample-lens distance. The size of the beam spot $w$ was determined by replacing the sample by a $10 \mu \mathrm{m}$ pinhole, or a $1 \mu \mathrm{m}$ pinhole in the latter case, and scanning across the beam (accuracy ca. 5\%). The glass cell was mounted in a sample holder and placed into an integrating sphere in order to average scattering intensities over all scattering angles of the transmitted light. A thick f ber bundle (diameter $5 \mathrm{~mm}$ ), positioned perpendicular to the incoming beam, was used to conduct the transmitted light from the integrating sphere to a photomultiplier. The f uctuations of the integrated transmission were analyzed using a commercial photon correlation setup. The detection limit for the intensity correlation function was determined to be lower than $10^{-6}$ over the whole range of correlation times $4 \times 10^{-7} s<\tau<10^{-5} s$ considered. Details of the experimental setup can be found in [18].

Fig. 1.5 shows the intensity-intensity correlation functions for three different beam spot sizes, with $l^{*}=0.98 \mu \mathrm{m}$. The maximum signal observed for this $\mathrm{f} \mathrm{lm}$ thickness $L=19.6 \mu m$ is of the order of $\langle I(0) I(0)\rangle /\langle I(0)\rangle^{2}-1 \approx 2 \times 10^{-4}$ corresponding to a conductance of $g \approx 5000$.

\subsection{INFLUENCE OF THE BOUNDARY LAYER}

We $\mathrm{f}$ rst want to explore the limits of $w \approx l^{*}$, where an increased inf uence of the light propagating in a layer near the sample surface is expected. In the case of $C_{2}$ correlations, crossing of light paths near the incoming surface results in short "active" scattering paths, after which no further dephasing occurs. These 


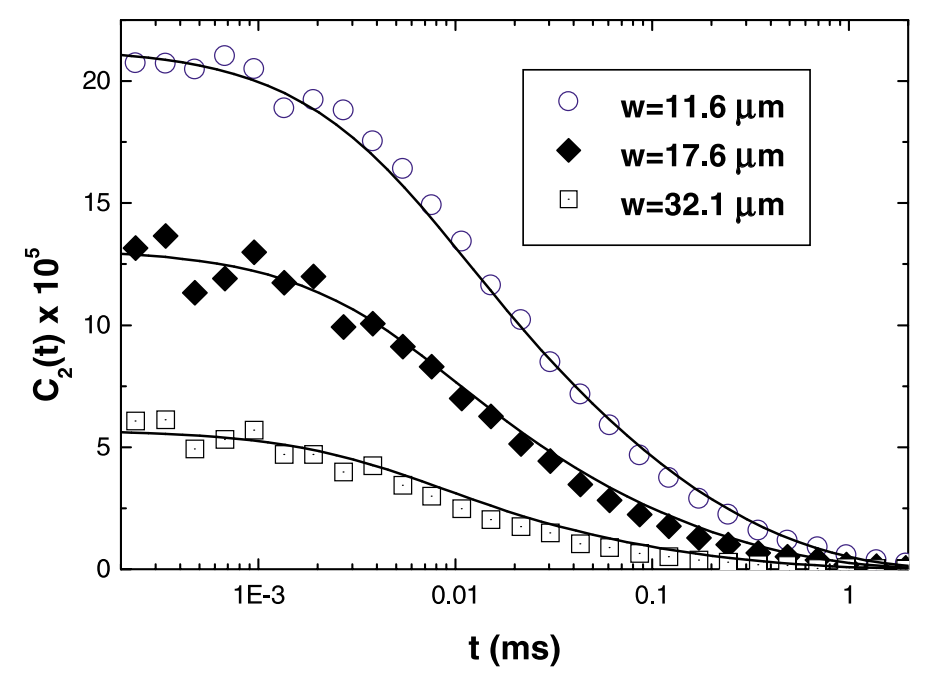

Figure 1.5 Dynamic long range correlation function $C_{2}(t)$ for a slab of thickness $L=19.6 \mu \mathrm{m}$ $\left[l^{*}=0.98 \mu \mathrm{m}\right]$. The amplitude $C_{2}$ increases with decreasing beam spot size $w$. The solid lines are calculated within the integral approximation, Eq. (1.22), with no adjustable parameter $\left(z_{0}=l^{*}\right)$.

short active paths are responsible for the long time tail of the correlation function $C_{2}(t)$ [see also section 3.4].

A signif cant inf uence of this boundary layer on the amplitude $C_{2}$ is expected when the beam size $w$ is of the order of the transport mean free path $l^{*}$, which is the length scale over which the incident light is randomized. Fig. 1.6 shows the dependence of $C_{2}^{-1}$ on $w$ for thick f $\operatorname{lms}(L=90 \pm 10 \mu \mathrm{m})$ of colloidal suspensions of different $l^{*}$. The concentration $\Phi$ of the suspensions is in all cases $11 \%$ or lower, therefore the refractive index $n \simeq n_{\text {water }} \simeq$ const. In this range, i.e. $w / L<0.2, C_{2}^{-1}$ scales in good approximation linearly with $w$ :

$$
C_{2}^{-1} \simeq \frac{8 \alpha}{5} w
$$

Eq. (1.18) can be derived by expanding Eq. (1.6) in the limit $w / L \rightarrow 0$. $C_{2}^{-1} \propto w$ follows also directly from the integral approximation [Eq. (1.13)] assuming diffuse linear spreading of the photon cloud inside the slab $w(z) \simeq$ $w(0)+\beta z$ with $\beta$ of order 1 [18].

In the experiments (Fig. 1.6) we clearly observe the linear dependence of $C_{2}^{-1}$ on $w$, however $C_{2}^{-1}$ does not tend to zero for small values of $w$, but reaches a well def ned minimum value $\left(1 / C_{2}\right)_{\min }$. Apparently, the light incident on the slab does not contribute to the long range correlations before it is scattered at least once inside a surface layer, hence broadening the beam spot [18]. This 


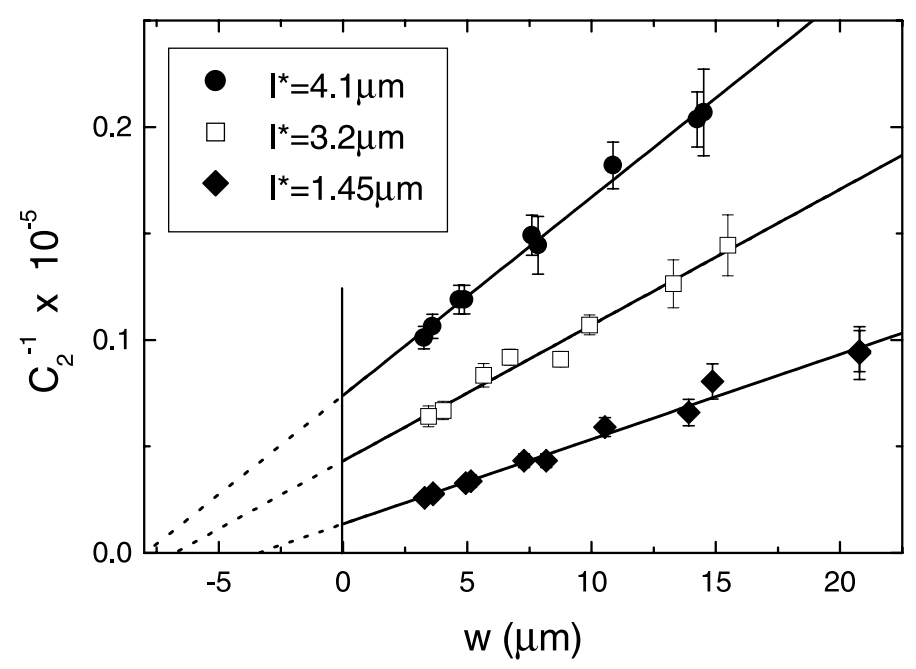

Figure 1.6 Measurements of $C_{2}$ vs. the beam spot size $w$ show that $C_{2}^{-1}$ values extrapolated for $w \rightarrow 0$ are non-zero and a distinct function of the transport mean free path $l^{*}$. A minimum beam spot size, $w_{\min }=2.4 l^{*}$, can be def ned by extrapolation of $C_{2}^{-1}(w) \rightarrow 0$ (dotted lines). The thickness of the $\mathrm{f} \operatorname{lms}(L=90 \pm 10 \mu \mathrm{m})$ is in all cases much larger than the beam spot size $w$.

result suggests that in fact the photon intensity distribution is broadened by scattering in a surface layer of thickness $(1-2) l^{*}$. We can account for this surface scattering by introducing an effective beam spot size:

$$
w_{\text {eff }}=\left(w_{\min }+w\right)=2.4 l^{*}+w
$$

We note that this value is somewhat larger than one would expect from single scattering contributions and also larger then the value determined from the shape of the correlation function (see section 3.4 ). Recent calculations suggest that $\mathrm{f}$ nite size effects may account for this discrepancy since they lead to an increase of $C_{2}^{-1}$ [31]. In the case $w / L \rightarrow 0$ the effective length $L_{\text {eff }}$ of the sample (where the correlations are built up) is of the order $L_{\text {eff }} \approx w$ which means $L_{e f f}$ is comparable to $l^{*}$ for small beamspot sizes $w$.

\subsection{AMPLITUDE SCALING OF $\boldsymbol{C}_{\mathbf{2}}$}

According to Eq. (1.6) the product $C_{2} \cdot L$ should depend solely on the ratio of the beam spot size and sample thickness $w / L$, independently of the actual values of $w$ and $L$. For this reason, the measured values of $\left(C_{2} L\right)^{-1}$ are predicted to follow a master curve. We expect the rescaled amplitude $\left(G_{2} L\right)^{-1}$ to increases linearly with $w / L$ for $w<<L$, whereas for large ratios $w / L>>$ 1 the quadratic dependence should be recovered: $\left(C_{2} L\right)^{-1} \sim(w / L)^{2}$. The 


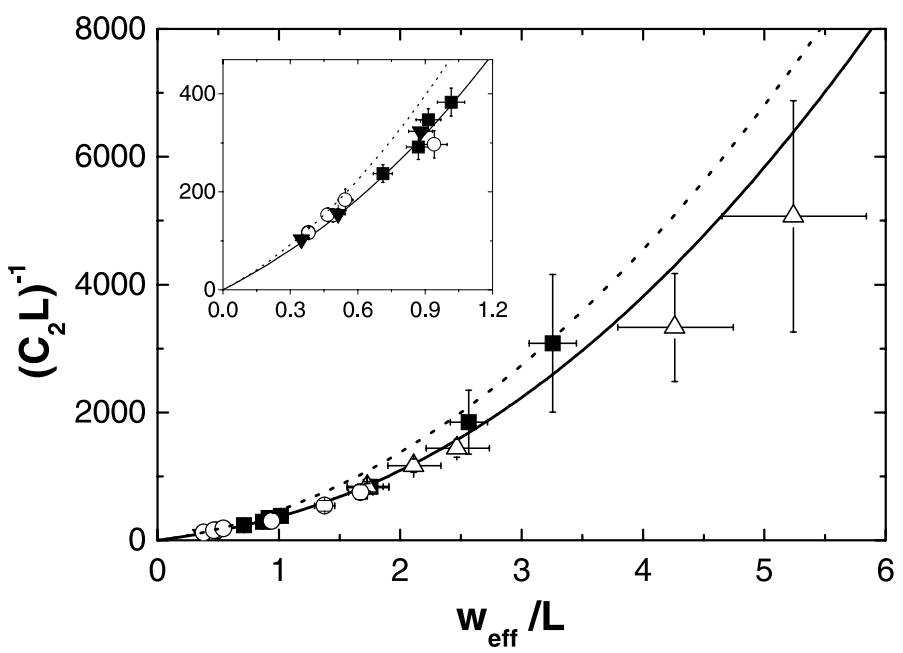

Figure 1.7 Scaling dependence of $\left(C_{2} L\right)^{-1}$ on the reduced beam spot size $w_{\text {eff }} / L$. The measured values for four different $\mathrm{f} l \mathrm{~m}$ thicknesses $L=8,19.6,36.7,40.2 \mu \mathrm{m}$ follow a master curve [Eq. (1.6), solid line] with $\alpha=149 \pm 15$ the only adjustable parameter. The integral approximation yields fairly good agreement with the same set of parameters [Eq. (1.21), dotted line].

amplitude $C_{2}$ for $\mathrm{flms}$ of different thicknesses $(L=8,19.6,36.7,40.2 \mu \mathrm{m})$ and effective beam spot sizes from 14 to about $60 \mu \mathrm{m}$ was determined for a single minimum transport mean free path of $l^{*}=0.98 \mu \mathrm{m}$. Over the explored range of $w_{e f f} / L$, the values $\left(C_{2} L\right)^{-1}$ are found to be in good agreement with the scaling prediction Eq. (1.6) (Fig. 1.7) with only one adjustable parameter $\alpha=149 \pm 15[1 / \mu \mathrm{m}]$. This value is in quantitative agreement with theory, $\alpha=138 \pm 27[1 / \mu m]$, and static measurements in the frequency domain [22].

Fairly good agreement with the same set of parameters is also obtained from Eq. (1.13) with the approximate intensity distribution $w(z) \simeq w_{e f f}+\beta z, \beta=$ $16 / 15[27,32]$ :

$$
\begin{gathered}
C_{2}(z)=\frac{2}{3 \alpha} \frac{1}{w(z)^{2}} \\
C_{2} L \simeq \int_{0}^{L} C_{2}(z) d z=\frac{2}{3 \alpha} \frac{1}{\left[\left(w_{e f f} / L\right)^{2}+\beta\left(w_{e f f} / L\right)\right]}
\end{gathered}
$$

Another feature is illustrated in Fig. 1.8. For $L / w_{\text {eff }}>>1$ the magnitude of the $C_{2}$ correlations becomes independent of $L$. This is due to the broadening of the beam inside the sample (Fig. 1.2). If $L$ is much larger than $w_{\text {eff }}$, the width of the photon cloud deep inside the sample becomes so large, that the 


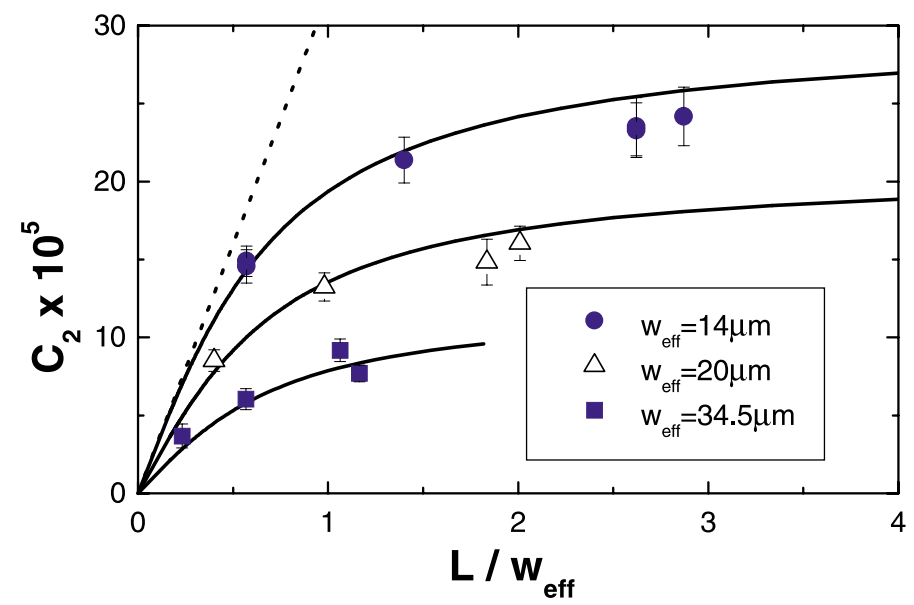

Figure 1.8 Dependence of $C_{2}$ on the slab thickness $L$. The values for three different beam spot sizes $w_{e f f}=14,20,34.5 \mu \mathrm{m}$ are plotted with the corresponding theoretical lines, Eq. (1.6), using $\alpha=149[1 / \mu \mathrm{m}]$. The dotted line shows the result for a corresponding cylindrical waveguide with $D=14 \mu m$ [Eq. (1.4)].

crossing probability is very small in most of the sample except the region of thickness of order $L_{e f f} \approx w_{\text {eff }}$ near to the entrance surface. Increasing the thickness yields only asymptotically small increases in $C_{2}$ (Fig. 1.8).

Localization of light. Since $1 / g \simeq C_{2}$ we f nd that for a slab geometry (by increasing $\mathrm{L}$ and decreasing $w$ ) the value of the dimensionless conductance $g$ cannot be reduced below a certain value $g_{\min }$. In fact the maximum amplitude $C_{2}$ or the minimal $g$ is determined by the transport mean free path [Eq. (1.18), Eq. (1.19)]: $g_{\min } \simeq(8 / 5) \alpha\left(2.4 l^{*}\right)=1.3\left(k_{0} l^{*}\right)^{2}[18]$.

The dimensionless conductance $\mathrm{g}$ is also an important quantity with respect to the transition from diffusion to localization of light. For a waveguide geometry $g<1$ implies a localization transition while the role of $g$ for the localization transition in a slab geometry is still under discussion.

From our experiments we extrapolate that $g<1$ can be achieved at $k l^{*} \approx 1$, a value that is of the same order as the Ioffe-Regel criterion for the localization transition: $k l^{*} \approx 1$ [33]. However for optical wavelengths $k \approx 15 / \mu m(\lambda / n \approx$ $400 \mathrm{~nm}$ ) this is only realized for (unphysically) small beamspot sizes $w \ll l^{*} \leq$ $1 / k_{0} \approx 70 \mathrm{~nm}[27]$.

Scaling with $l^{*}$. We were able to conf $\mathrm{rm}$ the predicted linear dependence of $C_{2}^{-1} \propto \alpha$ on the transport mean free path $l^{*}$ [Eq. (1.6)]. Fig. 1.9 shows the values of $\alpha$ determined from the slope of the $\left(C_{2}(w)\right)^{-1}$-curves (Fig. 1.6). A 


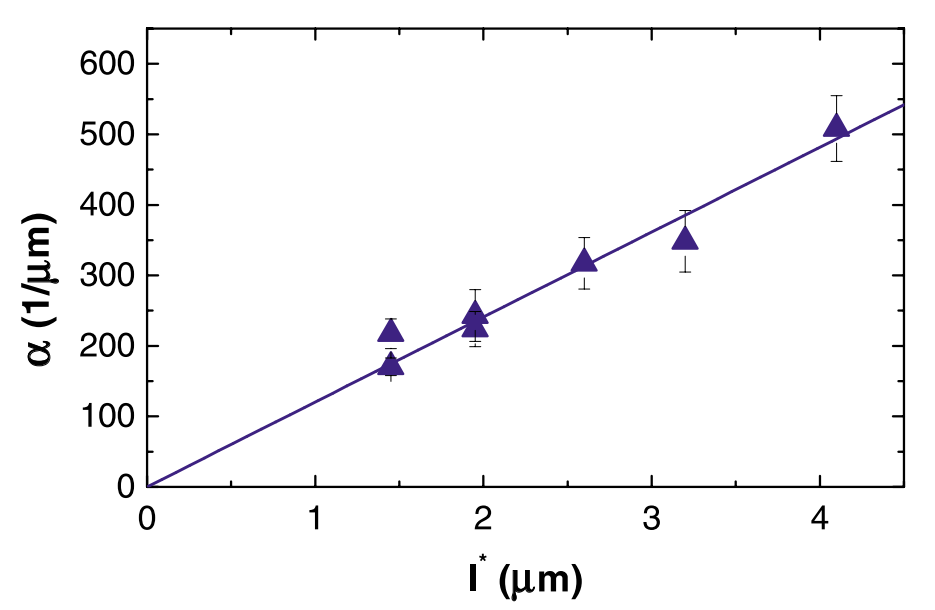

Figure 1.9 The values of $\alpha$ show the expected linear dependence on $l^{*}$. The $\alpha$ values are determined from the slope of measured $C_{2}^{-1}(w)$ curves (Fig. 1.6).

linear $\mathrm{ft}$ yields $\alpha / n^{2}=(71 \pm 9) * l^{*}$, compared to $\alpha / n^{2}=63 * l^{*}$ from theory [Eq. (1.7)].

\subsection{SHAPE OF THE CORRELATION FUNCTION}

Finally we want to discuss the time dependence of the correlation function $C_{2}(t)$. Crossing of light paths can occur at any point inside the sample, its probability being determined only by the effective lateral extension of the photon cloud. In the case of a cylindrical wave the crossing probability is independent of the depth $z$ which leads to Eq. (1.15), or equivalently Eq. (1.10). $C_{2}(t)$ therefore decays much slower and broader than $C_{1}(t)$. The semi-logarithmic plots in Fig. 1.3 and Fig. 1.10 clearly reveal this behavior.

For a complete description for any combination of $w$ and $L$ we use again the integral approximation for the correlation function $C_{2}(t)$ [Eq. (1.14)]. Using Eq. (1.21) with $w(z) \simeq w_{\text {eff }}+\beta z, \beta=16 / 15$ we f nd [27, 32]:

$$
C_{2}(t)=\frac{C_{2}}{L} \int_{z_{0}}^{L} \frac{2}{3 \alpha} \frac{1}{w(z)^{2}} \cdot \exp \left(-2\left(\frac{z}{l^{*}}\right)^{2} \frac{t}{\tau_{0}}\right) d z
$$

For the cylindrical waveguide limit, $w_{\text {eff }} \gg L$ and $z_{0}=0$, Eq. (1.22) reduces to Eq. (1.15) (see also Fig. 1.3). We furthermore introduced a lower bound $z_{0}$ for the integral which can be non-zero. This allows us to take into account single scattering contributions close to the boundary. In fact for long correlation times $C_{2}(t)$ does not show the expected algebraic decay $t^{-1 / 2}$ but 


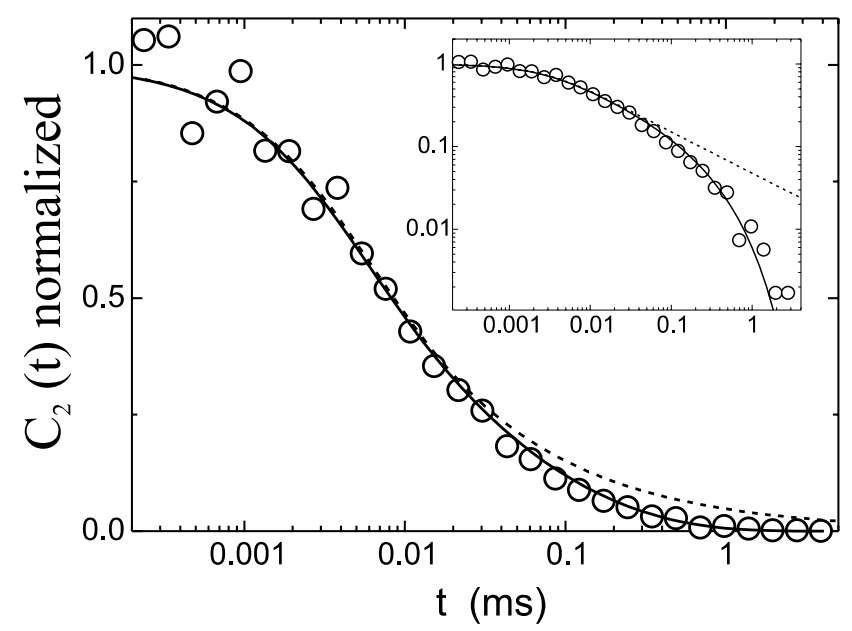

Figure 1.10 Normalized correlation function $C_{2}(t)$ for $w>L[L=19.6 \mu m, w=32 \mu m]$. A numerical $\mathrm{ft}$ with Eq. (1.10), dashed line, yields good overall agreement with $L=19.4 \mu \mathrm{m}$ in agreement with $L=19.6 \mu \mathrm{m}$ determined from measurements of $C_{1}(t)$. For long correlation times deviations from the predicted $t^{-1 / 2}$ behavior show up. We f nd perfect agreement (solid line) when introducing a lower bound $z_{0}=1.3 l^{*}$, in the integral approximation, Eq. (1.22). Inset: log-log plot of the same data set.

decays much faster [18]. The suggested explanation is that before a crossing of two light paths can take place there has to be at least one single scattering event close to the surface. This sets a lower bound to the minimum "active" path length. We take account for this by setting $z_{0} \simeq l^{*}$. In Fig. 1.5 and Fig. 1.10 it is shown that Eq. (1.22) perfectly describes the experiments over the whole range of correlation times both in the limit $w \ll L$ and $w \approx L$. The good agreement for longer correlation times gives further evidence for the suggested single scattering contributions.

\section{UNIVERSAL CONDUCTANCE FLUCTUATIONS OF LIGHT}

\subsection{THE EXPERIMENTAL REALIZATION}

The setup to measure $C_{3}(t)$, the optical analog of universal conductance f uctuations in disordered metals [13], is schematically displayed in Fig. 1.11. It was designed in analogy to a mesoscopic wire in two lead conf guration. The prelayer of variable thickness $L_{1}$ enables the separation of $C_{2}(t)$ and $C_{3}(t)$ in the time domain. The active path sections of $C_{2}(t)$ are located before the (single) crossing events which occur almost exclusively within the pinhole. A suff ciently thick prelayer $L_{1}$ therefore leads to a rapid decay of $C_{2}(t)$ very 


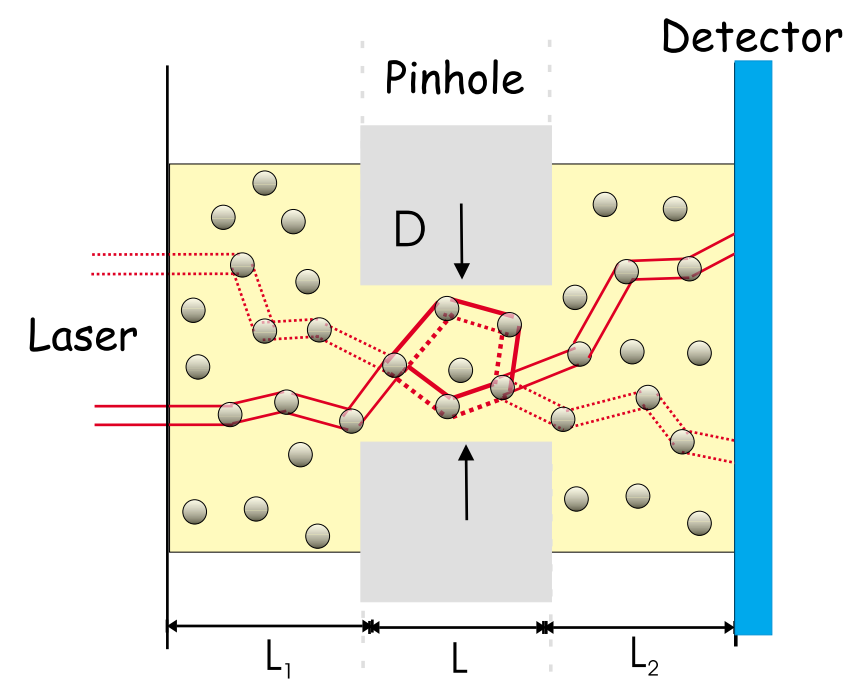

Figure 1.11 Sketch of the experimental setup. A small cylindrical pinhole (diameter $D$, length $L$ ) sandwiched between two layers $L_{1}$ and $L_{2}$ is f lled with a turbid colloidal suspension. Photon paths which cross twice inside the pinhole give rise to UCF.

similar to $C_{1}(t)$ for $L_{1} \gg L$, as can be seen when replacing the integration limits in Eq. (1.15) by $\left[L_{1}, L_{1}+L\right]$ :

$$
\begin{aligned}
C_{2}^{\prime}(t) & \simeq C_{2}^{\prime} \frac{1}{L} \int_{L_{1}}^{L_{1}+L} C_{1}(t, z) d z \\
& \simeq C_{2}^{\prime} \exp \left[-2\left(\frac{L_{1}}{l^{*}}\right)^{2} \frac{t}{\tau_{0}}\right] ; L_{1} \gg L
\end{aligned}
$$

To distinguish it from the broad and slow decay of $C_{2}(t)$ we call this rapidly decaying function $C_{2}^{\prime}(t) \simeq C_{1}\left(t, L_{1}\right)$. On the other hand, according to Eq. (1.16), $C_{3}(t)$ is expected to show an algebraic decay independent of the thickness of both layers $L_{1}$ and $L_{2}$ since the two crossing events occur essentially only within the pinhole. Physically the colloidal prelayer scrambles the incoming modes very rapidly thereby creating an effective multi-mode illumination of the pinhole on the time scales of interest for $C_{3}(t)$. 


\subsection{SETUP}

Samples were prepared from commercial colloidal $\mathrm{TiO}_{2}$ suspended in water. After stabilization with polyacrylic acid and fltering the suspension was found fairly monodisperse with an average particle diameter of $d \simeq 290 \mathrm{~nm}$, determined by single scattering photon correlation spectroscopy. Concentration was about $7 \%$ by volume. Using a cell of known thickness $(\mathrm{L}=100 \mu \mathrm{m})$ we f nd from DWS in transmission [Eq. (1.9)] $l^{*}=1.35 \pm 0.1 \mu \mathrm{m},\left(\tau_{0} \simeq 3 \mathrm{~ms}\right)$.

A cylindrical pinhole (laser drilled in a disc shaped stainless steel foil of thickness $L=13 \mu \mathrm{m}$ ) was embedded in the suspension providing a liquid reservoir on both sides of the sample. The thickness of both layers $\mathrm{L}_{1}, \mathrm{~L}_{2}$ sealed by glass windows was varied using different spacers. The sample was illuminated with a laser beam $(\lambda=514.5 \mathrm{~nm})$ focused down to $150-200 \mu \mathrm{m}$ beam diameter at intensities $<100 \mathrm{~mW}$. We performed measurements of the autocorrelation function of the angular integrated transmitted intensity collected with a thick multi-mode f ber (detector). Multiple runs of typically $500 \times 3$ $s e c$ were carried out at photon count rates of $500-2000 \mathrm{kHz}$. In this geometry the contribution $C_{1}(t)$ is time independent due to the angular averaging of the outgoing light over many speckle spots.

\subsection{LONG RANGE CORRELATIONS}

We characterized the setup by measuring $C_{2}^{\prime}(t)$ for different pinhole sizes using moderately thick surrounding layers $\left(L_{1}, L_{2} \approx 50 \mu \mathrm{m}\right)$. The inset in Fig. 1.12 shows the $C_{2}^{\prime}$ values determined from the amplitude of $C_{2}^{\prime}(t)$. For a quantitative comparison with theory it is necessary to take also into account contributions outside the pinhole where the effective lateral conf nement of photon cloud spreads out linearly. We can write:

$$
C_{2}^{\prime} \simeq \sum_{i=1}^{2} C_{2}^{i}
$$

The contributions inside the pinhole are given by the expression for a cylindrical waveguide $C_{2}^{1}=(4 L) /\left(k_{0}^{2} D^{2} l^{*}\right)$, Eq. (1.4), whereas the contributions outside the pinhole are due to an intensity step prof le spreading out in a semi-inf nite sample $C_{2}^{2}=3 /\left(2 k_{0}^{2} D l^{*}\right)$, Eq. (1.5), hence

$$
C_{2}^{\prime} \simeq \frac{4}{k_{0}^{2} D^{2}} \frac{L}{l^{*}}+\frac{3}{2 k_{0}^{2} D l *}=\frac{4}{k_{0}^{2} D^{2}} \frac{L+(3 / 8) D}{l^{*}}
$$

Hence we can take account for this additional contribution by introducing an effective length of the pinhole $L+(3 / 8) D$.

The experimental results are in excellent agreement with this prediction. It can be readily seen that due to the quadratic dependence of $C_{2}$ on $D$ the expected 

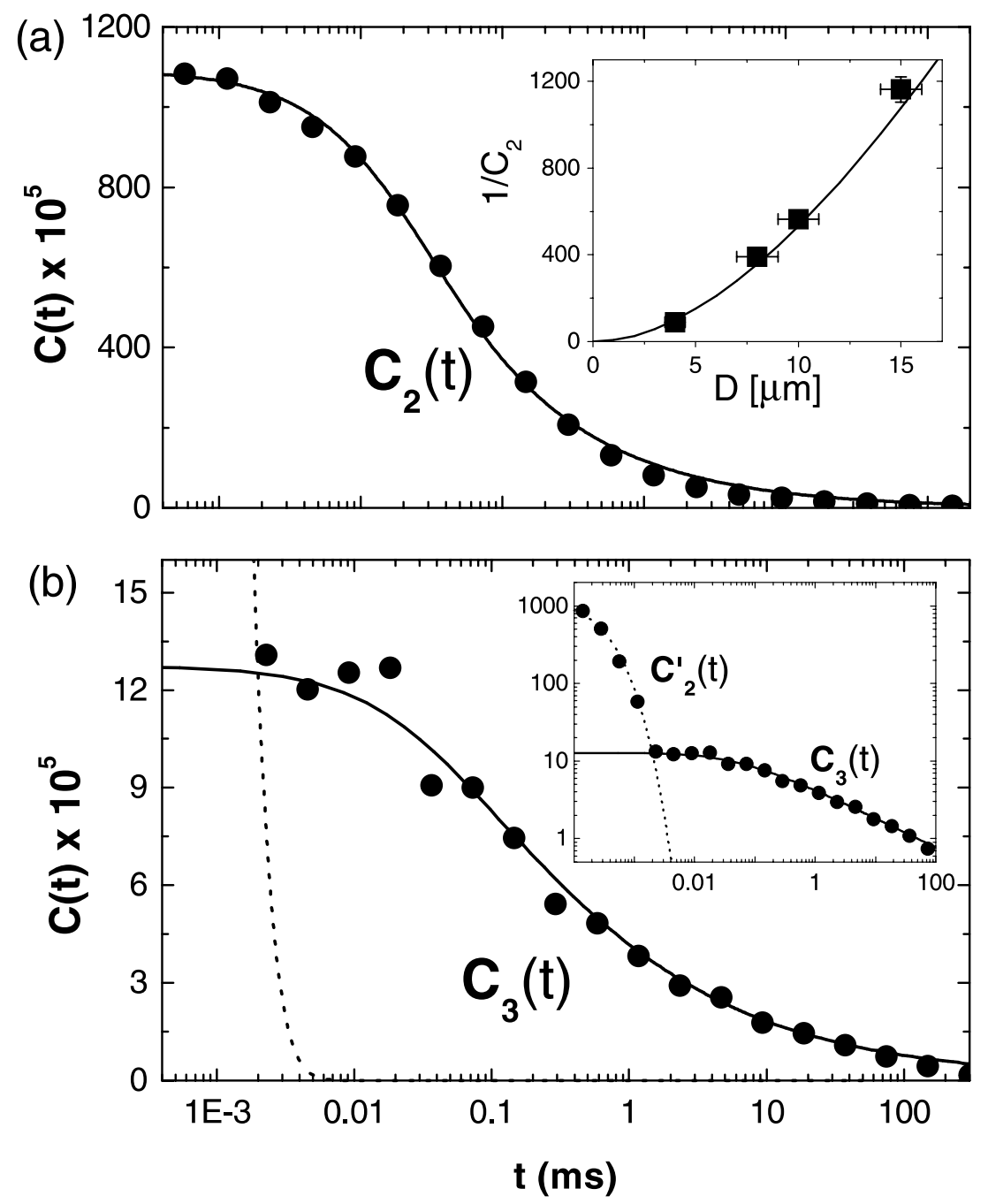

Figure 1.12 (a) Inset: Inverse amplitude $1 / C_{2}^{\prime}$ as a function of the pinhole diameter $D$. Solid line: theoretical prediction, Eq. (1.25), with no adjustable parameter. Main f gure: $C_{2}(t)$ correlation function for the smallest pinhole $D=4 \mu \mathrm{m}$ (full circles). Solid line: best $\mathrm{ft}$ by Eq. (1.15) with $C_{2}=1.1 \cdot 10^{-2}, L=13.1 \pm 1.3 \mu \mathrm{m}$. (b) Universal conductance f uctuations $C_{3}(t)$ in comparison with $C_{2}^{\prime}(t)$. For $t>2 \times 10^{-3} \mathrm{~ms}, C_{2}^{\prime}(t)$ (dotted line) has decayed and $C_{3}(t)$ clearly shows up. Solid line: theory [Eq. (1.16)] with $C_{3}=1.3 \cdot 10^{-4}, L=13.1 \mu \mathrm{m}$. Inset: log-log plot of the same data set. 
amplitude of $C_{3}(t)$ is very small, $C_{3} \approx\left(C_{2}^{\prime}\right)^{2}<10^{-5}$, for all pinhole sizes except for the smallest one $D=(4 \pm 0.5) \mu m$.

\subsection{OBSERVATION OF OPTICAL UCF}

To achieve an effective separation of time scales between $C_{2}(t)$ and $C_{3}(t)$ we used a prelayer thickness $L_{1} \approx 100 \mu \mathrm{m}$. To increase transmission, we replaced the colloidal suspension in $\left(L_{2}\right)$ by pure water. Due to the absence of scattering in $L_{2}$ we were now able to measure the full $C_{2}(t)$ correlation function [Eq. (1.15)] of a cylindrical waveguide by illuminating the sample from the $L_{2}$-side. This provides additional information about the dynamics of the particles inside the pinhole which is diff cult to obtain otherwise. The measured correlation function is shown in Fig. 1.12. Since we expect $C_{2}=$ $(1 \pm 0.2) \cdot 10^{-2} \approx 1 / \mathrm{g}$ from theory for the pinhole foil thickness $L=13 \mu \mathrm{m}$, it is in excellent agreement with the theoretical prediction in amplitude, shape and characteristic decay time. These results demonstrate that the dynamics of the particles inside the pinhole are largely unaffected by the lateral conf nement and that the distribution of path lengths is not signif cantly altered by residual absorption at the pinhole walls.

In order to measure UCF the identical sample $(D=4 \mu \mathrm{m})$ was used which was now simply illuminated from the opposite side $\left(L_{1}\right)$. As seen in Fig. 1.12, the contribution of $C_{2}^{\prime}(t)$ now decays very fast. For longer correlation times we observe $C_{3}(t)$ which decays over more than four decades in time. The amplitude $C_{3} \simeq 1.3 \cdot 10^{-4}$ is found in good agreement with the value $C_{2}=1.1 \cdot 10^{-2}$ obtained from the $C_{2}(t)$ measurement. Equally good agreement is found by comparing the shape of the $C_{3}(t)$ correlation function with the theoretical curve [Eq. (1.16)] without any adjustable parameter.

\section{SUMMARY AND CONCLUSIONS}

It has been shown that the use of coherent laser sources combined with accurate time correlation techniques allows to study very precisely the higher order correlation functions $C_{2}(t)$ and $C_{3}(t)$. Based on a series of measurements with different sample thicknesses $L$, beam spot sizes $w$, and transport mean free paths $l^{*}$, it has been possible to quantitatively conf rm the scaling predictions for $C_{2}$. The time dependent correlation function $C_{2}(t)$ shows a good overall agreement with diagrammatic calculations. However the predicted long time $t^{-1 / 2}$ algebraic tail has not been observed. Quantitative agreement can be achieved by introducing a cut-off for the contribution of short scattering paths to $C_{2}(t)$. The study of the amplitude $C_{2}$ for extremely small values of $w \approx l^{*}$ delivers further evidence that light has to be scattered at least once before correlations can be built up by crossing of light paths. 
Universal conductance fuctuations (UCF) in the transmission of classical waves have been observed using very small samples of concentrated colloidal suspensions. The experimental results provide a complete picture of the microscopic origin of UCF in disordered conductors in general. This demonstrates that the (quantum) wave interference can be quantitatively described by the simple model of diffusing waves crossing at locations inside the sample where the lateral conf nement is high. Like weak and strong Anderson localization, $\mathrm{UCF}$ are a direct consequence of wave interference effects on a macroscopic scale. These interference corrections increase with randomness resulting in the breakdown of classical transport theory.

\section{Acknowledgments}

F.S. would like to thank Juanjo Saenz for useful comments and many stimulating discussions.

\section{References}

[1] P.W. Anderson, Phil. Mag. 52505 (1985); S. John, Phys. Rev. B 31304 (1985)

[2] S. John, Physics Today, May 1991, p.32-40

[3] M.P. van Albada and A. Lagendjik, Phys. Rev. Lett. 55, 2692 (1985)

[4] P.E. Wolf and G. Maret, Phys. Rev. Lett. 55, 2696, (1985)

[5] P. Sheng (Ed.): Scattering and localization of classical waves in random media, World Scientif c, Singapore 1990, Ping Sheng,Introduction to Wave Scattering, Localization, and Mesoscopic Phenomena, Academic Press, Boston, 1995.

[6] C.M. Soukoulis (Ed.): Photonic band gaps and localization, Nato ASI Series B, Physics 308 (Plenum, N.Y., 1993).

[7] G. Maret in: Mesoscopic Quantum Physics, E. Akkermans, G. Montambaux, J-L. Pichard and J. Zinn-Justin, eds (Elsevier Science B.V., North Holland, 1995), p.147

[8] D. S. Wiersma, P. Bartolini, A. Lagendijk and R. Righini, Nature 390, 671 (1997)

[9] F. Scheffold, R. Lenke, R. Tweer and G. Maret, Nature 398, 206 (1999)

[10] F.J.P. Schuurmans, M. Megens, D. Vanmaekelbergh and A. Lagendijk, Phys. Rev. Lett 83, 2183 (1999)

[11] S. Feng, C. Kane, P.A. Lee and A.D. Stone, Phys. Rev. Lett. 61, 834 (1988)

[12] R. Pnini and B. Shapiro, Phys. Rev. B 39 , 6986 (1989)

[13] S. Feng and P.A. Lee, Science 251, 633 (1991)

[14] R. Berkovits and S. Feng, Phys. Rep. 238, 135(1994) 
[15] M.C.W. van Rossum and T. M. Nieuwenhuizen, Rev. Mod. Phys. 71,313 (1999)

[16] A.Z. Genack, N. Garcia, W. Polkosnik, Phys. Rev. Lett. 65, 2129 (1990)

[17] M.P. van Albada, J.F.de Boer and A.Lagendijk, Phys. Rev. Lett. 64, 2787 (1990)

[18] F. Scheffold, W. Härtl, G. Maret and E. Matijević, Phys. Rev. B 56, 10942 (1997)

[19] F. Scheffold and G. Maret, Phys. Rev. Lett. 81, 5800 (1999)

[20] C.P. Umbach et al., Phys. Rev. B. 304048 (1984); R.A. Webb et al., Phys. Rev. Lett. 542696 (1985)

[21] This differs by a factor of 2 from the original results for scalar waves [12] due to the two polarization states of electromagnetic waves [22].

[22] J.F. de Boer, M.P. van Albada and A. Lagendijk, Phys. Rev. B 45, 658 (1992)

[23] G. Maret and P.E. Wolf, Z. Phys. B 65409 (1987); D.J. Pine, D.A. Weitz, P.M. Chaikin and E. Herbolzheimer, Phys. Rev. Lett. 601134 (1988); D.A. Weitz and D.J. Pine in Dynamic Light Scattering, W. Brown Ed. (Oxford Univ.Press, New York, 1993), pp 652-720

[24] P.D. Kaplan, M.H. Kao, A.G. Yodh and D.J. Pine, Appl. Opt. 32, 21, 3828(1993)

[25] G. Maret, Curr. Opin. Coll. Int. Sci. 2, 251-257 (1997)

[26] S. Romer, F. Scheffold and P. Schurtenberger, submitted to Phys. Rev. Lett.

[27] F. Scheffold and G. Maret, in preparation

[28] F. Scheffold, PhD thesis, University of Konstanz (1998)

[29] We note that a $\mathrm{f}$ rst digrammatic calculation of $C_{3}(t)$ has been published by Berkovits and Feng [14] but we were not able to reproduce their plots which are based on a complex combination of different diverging functions. Recently van Rossum and Nieuwenhuizen have presented an new calculation of $C_{3}(t)$ which has not been compared yet to the results of our simple integral approximation, Eq. (1.16) [15]. Attemps are now underway to do so [27].

[30] Y-S. Her, E. Matijević and M.C. Chon, J. Mater. Res. 10, 12 , 3106 (1995)

[31] A. Garcia-Martin, F. Scheffold, M. Nieto-Vesperinas and J.J. Saenz, in preparation

[32] The prefactor to Eq. (1.12) and the value of $\beta=16 / 15$ where determined by comparing the limiting cases $[w \ll L, w \gg L]$ to the results of Eq. (1.6). 
[33] Mott, N.F. Metal Insulator Transitions (Taylor and Franzis, London, 1974) 